Geometry $\&$ Topology

Volume 5 (2001) 521-550

Published: 21 May 2001

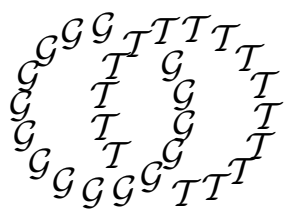

\title{
Metric characterizations of spherical and Euclidean buildings
}

\author{
Ruth Charney \\ AleXANDER LyTCHAK \\ Mathematics Department, Ohio State University \\ 231 W 18th Ave, Columbus, OH 43210, USA \\ and \\ Mathematisches Institut der Universität Bonn \\ Wegelerstraße 10, D-53115 Bonn, Germany \\ Email: charney@math.ohio-state.edu and lytchak@math.uni-bonn.de
}

\begin{abstract}
A building is a simplicial complex with a covering by Coxeter complexes (called apartments) satisfying certain combinatorial conditions. A building whose apartments are spherical (respectively Euclidean) Coxeter complexes has a natural piecewise spherical (respectively Euclidean) metric with nice geometric properties. We show that spherical and Euclidean buildings are completely characterized by some simple, geometric properties.
\end{abstract}

AMS Classification numbers Primary: 20E42

Secondary: 20F65

Keywords: Buildings, CAT(0) spaces, spherical buildings, Euclidean buildings, metric characterisation

Proposed: Walter Neumann

Seconded: Jean-Pierre Otal, Steve Ferry

(C) Geometry $8 \mathcal{T}$ Topology Publications
Received: 23 November 2000

Revised: 11 May 2001 


\section{Introduction}

In recent years, much attention has been given to curvature properties of piecewise Euclidean and piecewise spherical complexes. A notion of curvature bounded above for such complexes was introduced by Alexandrov in the 1950's and further developed in the 1980's by Gromov. The curvature bound is defined as a condition on the shape of triangles (they must be sufficiently "thin") and is known as a CAT-inequality (Comparison inequality of AlexandrovToponogov). Some particularly nice examples of spaces satisfying CAT-inequalities are spherical and Euclidean buildings which come equipped with a natural piecewise spherical or Euclidean metric.

Buildings also satisfy other nice metric properties. A spherical building $X$, for example, is easily seen to have diameter $\pi$, as does the link of any simplex in $X$. It is natural to ask whether Euclidean and spherical buildings are characterized by their metric properties. In this paper, we give several metric characterizations of buildings. For example we prove

Theorem Let $X$ be a connected, piecewise spherical (respectively Euclidean) complex of dimension $n \geq 2$ satisfying

(1) $X$ is $C A T(1)$ (respectively CAT(0)).

(2) Every $(n-1)$-cell is contained in at least two $n$-cells.

(3) Links of dimension $\geq 1$ are connected.

(4) Links of dimension 1 have diameter $\pi$.

Then $X$ is isometric to a spherical building (respectively a metric Euclidean building).

Metric Euclidean buildings are products of irreducible Euclidean buildings, cones on spherical buildings, trees, and nonsingular Euclidean spaces (see Section 5).

Another metric property of buildings is that for every local geodesic $\gamma$, the set of directions in which $\gamma$ can be geodesically continued is non-empty and discrete. We call this the "discrete extension property".

Theorem Let $X$ be a connected, piecewise spherical (respectively Euclidean) complex of dimension $n \geq 2$ satisfying

(1) $X$ is $C A T(1)$ (respectively $C A T(0)$ ).

(2) $X$ has the discrete extension property.

Then $X$ is isometric to a spherical building (respectively metric Euclidean building). 
Werner Ballmann and Michael Brin, studying the question of rank rigidity for piecewise Euclidean complexes of nonpositive curvature, have obtained related results in [2] and [3], including a metric characterization of spherical and Euclidean buildings of dimension 2. Bruce Kleiner has also described (unpublished) a metric characterization of Euclidean buildings under the assumption that every geodesic is contained in an $n$-flat.

The first two sections of the paper contain background about buildings and geodesic metric spaces. The key problem in identifying a building is the construction of enough apartments. Sections 3, 4, and 5 are devoted to this task. Section 6 considers the 1-dimensional case, and Section 7 combines these results to arrive at the main theorems.

The first author would like to thank Bruce Kleiner for helpful conversations. The second author is indebted to Werner Ballmann for suggesting the question and for his continued support during the development of this paper. The first author was partially supported by NSF grant DMS-9803374.

\section{Buildings}

In this section we review some definitions and terminology. For more details about buildings, see [6] and [5].

Let $S$ be a finite set. A Coxeter matrix on $S$ is a symmetric function $m: S \times S \rightarrow$ $\{1,2, \ldots, \infty\}$ such that $m(s, s)=1$ and $m(s, t) \geq 2$ for $s \neq t$. The Coxeter group associated to $m$ is the group $W$ given by the presentation

$$
W=\left\langle S \mid(s t)^{m(s, t)}=1, s, t \in S\right\rangle .
$$

The pair $(W, S)$ is called a Coxeter system. If $T \subset S$, then the subgroup $W_{T}$ generated by $T$ is the Coxeter group associated to $\left.m\right|_{T \times T}$. The Coxeter system $(W, S)$ is irreducible if there is no non-trivial partition $S=S_{1} \amalg S_{2}$ such that $W=W_{S_{1}} \times W_{S_{2}}$.

The Coxeter group $W$ can be realized as a discrete group of linear transformations of an $n$-dimensional vector space $V$, with the generators $s \in S$ acting as reflections across the walls of a simplicial cone. This action preserves a bilinear form $B$ on $V$ represented by the matrix $B(s, t)=-\cos \left(\frac{\pi}{m(s, t)}\right)$ (where $\frac{\pi}{\infty}$ is taken to be 0$). W$ is finite if and only if this form is positive definite. In this case, $W$ acts as a group of orthogonal transformations and the action restricts to the unit sphere $S(V)$ in $V$. Hence $W$ is called a spherical Coxeter group.

If $B$ is positive semi-definite (but not definite) and $W$ is irreducible, then the action of $W$ on $V$ induces an action on an (n-1)-dimensional affine space 
$\mathbb{R}^{n-1}=V / V^{\perp}$ with the generators acting as affine reflections across the walls of a simplex. In this case, $W$ is called an irreducible Euclidean Coxeter group. A key fact about irreducible Euclidean Coxeter groups is that for any proper subgroup $T \subset S, W_{T}$ is a spherical Coxeter group. More generally, we call $W$ a Euclidean Coxeter group if it is a direct product of irreducible Euclidean Coxeter groups.

To any Coxeter group $W$, one can associate a simplicial complex $\Sigma_{W}$ called the Coxeter complex for $W$. In the case of spherical and Euclidean Coxeter groups, the Coxeter complex has a simple, geometric description. Let $M=$ $\mathbb{S}^{n-1}=S(V)$ if $W$ is spherical or $M=\mathbb{R}^{n-1}=V / V^{\perp}$ if $W$ is irreducible Euclidean. For each element $r \in W$ which acts as a reflection on $M$ (namely, $r$ is a generator or conjugate of a generator), $r$ fixes some hyperplane, called a wall of $M$. The walls divide $M$ into simplices. The resulting simplicial complex, $\Sigma_{W}$, is the Coxeter complex for $W$. If $W$ is a product of irreducible Euclidean Coxeter groups, then $\Sigma_{W}$ is the product of the corresponding Coxeter complexes. The top dimensional simplices (or cells) of $\Sigma_{W}$ are called chambers. $W$ acts freely transitively on the set of chambers of $\Sigma_{W}$ and the stabilizer of any lower dimensional cell $\sigma$ is conjugate to $W_{T}$ for some $T \subset S$.

There are several equivalent definitions of buildings. The most convenient for our purposes is the following (see [6]).

Definition 1.1 A building is a simplicial complex $X$ together with a collection of subcomplexes $\mathcal{A}$, called apartments, satisfying

(1) each apartment is isomorphic to a Coxeter complex,

(2) any two simplices of $X$ are contained in a common apartment,

(3) if two apartments $A_{1}, A_{2}$ share a chamber, then there is an isomorphism $A_{1} \rightarrow A_{2}$ which fixes $A_{1} \cap A_{2}$ pointwise.

If, in addition, every codimension 1 simplex is contained in at least three chambers, then $X$ is a thick building. It follows from conditions (2) and (3) that all of the apartments are isomorphic to the same Coxeter complex $\Sigma_{W}$. We say that a building $X$ is spherical (respectively Euclidean) if $W$ is spherical (respectively Euclidean).

Although the collection of apartments $\mathcal{A}$ is not, in general unique, there is a unique maximal set of apartments. We will always assume $\mathcal{A}$ to be maximal.

If $X_{1}, X_{2}$ are spherical buildings with associated Coxeter groups $W_{1}, W_{2}$, then the join $X_{1} * X_{2}$ is a spherical building with Coxeter group $W_{1} \times W_{2}$. In particular, the suspension $\Sigma X_{2}=\mathbb{S}^{0} * X_{2}$ is a building with Coxeter group 
$\mathbb{Z} / 2 \times W_{2}$. (Note that for $k>0$, the simplicial structure on $\mathbb{S}^{k} * X_{2}$ depends on a choice of identification of $\mathbb{S}^{k}$ with the $(\mathrm{k}+1)$-fold join $\mathbb{S}^{0} * \cdots * \mathbb{S}^{0}$. Despite this slight ambiguity, we will consider the $k$-fold suspension of a building to be a building.) Conversely, if $X$ is a spherical building whose Coxeter group $W$ splits as a product $W_{1} \times W_{2}$, then $X$ can be decomposed as the join of a building for $W_{1}$ and a building for $W_{2}$ (see [13], Theorem 3.10). Similarly, any Euclidean building splits as a product of irreducible Euclidean buildings.

\section{Metrics}

A metric space $(X, d)$ is a geodesic metric space if for any two points $x, y \in X$, there is an isometric embedding $\gamma:[0, a] \rightarrow X$ with $\gamma(0)=x$ and $\gamma(a)=y$. Such a path is called a geodesic segment or simply a geodesic from $x$ to $y$. An isometric embedding of $\mathbb{R}$ into $X$ is also called a geodesic, and an isometric embedding of $[0, \infty)$ is called a ray.

A piecewise Euclidean (respectively piecewise spherical) complex is a polyhedral cell complex $X$ together with a metric $d$ such that each cell of $X$ is isometric to a convex polyhedral cell in $\mathbb{R}^{n}$ (respectively $\mathbb{S}^{n}$ ) for some $n$, and

$$
d(x, y)=\inf \{\operatorname{length}(\gamma) \mid \gamma \text { is a path from } x \text { to } y\}
$$

for any $x, y \in X$. We will also assume that the metric $d$ is a complete, geodesic metric. In particular, the infimum $d(x, y)$ is realized by the length of some path $\gamma$.

If $X$ is a piecewise spherical or Euclidean complex and $x$ is a point in $X$, then the set of unit tangent vectors to $X$ at $x$ is called the link of $x$ and denoted $\mathrm{lk}(x, X)$. It comes equipped with the structure of a piecewise spherical complex, since the link of $x$ in a single $n$-cell is isometric to a polyhedral cell in $\mathbb{S}^{n-1}$. If $\sigma$ is a $k$-cell in $X$, we define $\mathrm{lk}(\sigma, X)$ to be the set of unit tangent vectors orthogonal to $\sigma$ at any point $x$ in the relative interior of $\sigma$. This set also has a natural piecewise spherical structure and we can identify

$$
\operatorname{lk}(x, X)=\operatorname{lk}(x, \sigma) * \operatorname{lk}(\sigma, X)=\mathbb{S}^{k-1} * \operatorname{lk}(\sigma, X)
$$

where the joins $*$ are orthogonal joins in the sense of [7]. (See [4] or the appendix of [7] for a discussion of joins of piecewise spherical complexes.)

In some cases, we may wish to consider spaces which do not have a globally defined cell structure. For this, we introduce the notion of a locally spherical space of dimension $n$. The definition is inductive. A locally spherical space of dimension 0 is a nonempty disjoint union of points. A locally spherical (respectively Euclidean) space of dimension $n, n>0$, is a complete geodesic 
metric space $(X, d)$ for which every point $x$ has a neighborhood isometric to a spherical (respectively Euclidean) cone on a locally spherical space $L_{x}$ of dimension $n-1$. We call such a neighborhood a conelike neighborhood of $x$. Clearly, $L_{x}=\mathrm{lk}(x, X)$. A piecewise spherical (respectively Euclidean) complex is a locally spherical (respectively Euclidean) space of dimension $n$ if and only if every cell is contained in an $n$-dimensional cell.

The basis for our metric characterization of buildings will be the CAT-inequalities defined by Gromov in [9]. Let $(X, d)$ be a complete, geodesic metric space and let $T$ be a geodesic triangle in $X$. A Euclidean comparison triangle for $T$ is a triangle $T^{\prime}$ in $\mathbb{R}^{2}$ with the same side lengths as $T$. We say $X$ is a $C A T(0)$ space if every geodesic triangle $T$ is "thin" relative to its comparison triangle $T^{\prime}$. That is, given any points $x, y \in T$, the distance from $x$ to $y$ in $X$ is less than or equal to the distance in $\mathbb{R}^{2}$ between the corresponding points $x^{\prime}, y^{\prime} \in T^{\prime}$. We define a $C A T(1)$ space similarly by comparing geodesic triangles $T$ in $X$ with spherical comparison triangles $T^{\prime}$ in $\mathbb{S}^{2}$. In this case, however, we only require the thinness condition to hold for triangles $T$ of perimeter $\leq \pi$ (since no comparison triangle exists with perimeter $>\pi$ ).

In the next two theorems we collect some facts about CAT(0) and CAT(1) spaces. These are due to Gromov, Ballmann, Bridson and others. A good source of proofs is [4] or [1].

Theorem 2.1 Let $X$ be a piecewise (or locally) Euclidean geodesic metric space.

(1) $X$ is locally $C A T(0)$ if and only if $l k(\sigma, X)$ is $C A T(1)$ for every cell $\sigma$.

(2) $X$ is $C A T(0)$ if and only if it is locally $C A T(0)$ and simply connected.

(3) If $X$ is $C A T(0)$, then any two points in $X$ are connected by a unique geodesic and any path which is locally geodesic, is geodesic.

Theorem 2.2 Let $X$ be a piecewise (or locally) spherical geodesic metric space.

(1) $X$ is locally $C A T(1)$ if and only if $l k(\sigma, X)$ is CAT(1) for every cell $\sigma$.

(2) $X$ is $C A T(1)$ if and only if it is locally CAT(1), any two points of distance $<\pi$ are connected by a unique geodesic, and these geodesics vary continuously with their endpoints.

(3) If $X$ is $C A T(1)$, then any path of length $\leq \pi$ which is locally geodesic, is geodesic. 
A Euclidean (respectively spherical) building $X$ of dimension $n$ comes equipped with a natural piecewise Euclidean (respectively piecewise spherical) metric in which each apartment is isometric to $\mathbb{R}^{n}$ (respectively $\mathbb{S}^{n}$ ) with the Coxeter group $W$ acting by isometries. In the spherical case, there is a unique such metric. In the Euclidean case, this metric is determined only up to scalar multiple on each irreducible factor. In this paper, for a piecewise Euclidean (respectively spherical) complex $(X, d)$, the statement that $X$ is a Euclidean (respectively spherical) building will mean that, the cell structure on $X$ satisfies the conditions of Definitions 1.1 and that the metric on $X$ is the natural building metric.

Define the diameter of $X$ to be

$$
\operatorname{diam}(X)=\sup \{d(x, y) \mid x, y \in X\} .
$$

The natural metric on a building satisfies a number of nice properties which are described in the proposition below.

Proposition 2.3 Let $X$ be a Euclidean (respectively spherical) building of dimension $n$ with the natural metric. Then:

(1) $X$ is $C A T(0)$ (respectively $X$ is $C A T(1)$ and daim $(X)=\pi$ ).

(2) For any simplex $\sigma$ of codimension $\geq 2, \operatorname{lk}(\sigma, X)$ is a spherical building. In particular, $l k(\sigma, X)$ is $C A T(1)$ and $\operatorname{diam}(\operatorname{lk}(\sigma, X))=\pi$.

(3) A subspace $A \subset X$ is an apartment if and only if the intrinsic metric on $A$ is isometric to $\mathbb{R}^{n}$ (respectively $\mathbb{S}^{n}$ ). Moreover, the inclusion $A \hookrightarrow X$ is an isometric embedding.

Proof (1) and (2) are well known. (1) follows from the fact that every geodesic in $X$ is contained in an apartment (see [8] or [6]). (2) follows from the fact that the isotropy group of a simplex $\sigma$ in $\Sigma_{W}$ is a spherical Coxeter group $W_{\sigma}$. The action of $W_{\sigma}$ on the sphere $\operatorname{lk}\left(\sigma, \Sigma_{W}\right)$ gives a natural identification of lk $\left(\sigma, \Sigma_{W}\right)$ with the Coxeter complex for $W_{\sigma}$. These constitute the apartments of $\operatorname{lk}(\sigma, X)$.

For (3), let $M=\mathbb{R}^{n}$ if $X$ is Euclidean and $M=\mathbb{S}^{n}$ if $X$ is spherical. Consider the collection of subspaces

$$
\mathcal{A}=\{A \subset X \mid A \text { is isometric to } M\} .
$$

By definition of the metric on $X, \mathcal{A}$ contains all the apartments of $X$. Since we are assuming the set of apartments to be maximal, it suffices to show that $\mathcal{A}$ satisfies the conditions in Definition 1.1 for a system of apartments. 
Observe first that any subspace $A$ isometric to $M$ is necessarily a subcomplex of $X$ since its intersection with any $n$-simplex $\sigma$ must be both open and closed in $\sigma$. By induction on the dimension of $X$, we may assume that for any simplex $\sigma \subset A, \operatorname{lk}(\sigma, A) \hookrightarrow \mathrm{k}(\sigma, X)$ is an isometric embedding. It follows that the embedding $A \hookrightarrow X$ preserves local geodesics. If $X$ is Euclidean, then it is $\operatorname{CAT}(0)$, hence local geodesics are geodesics. If $X$ is spherical, then it is $\mathrm{CAT}(1)$, hence local geodesics of length $\leq \pi$ are geodesics. In either case, we conclude that $A \hookrightarrow X$ maps geodesics to geodesics, so it is an isometric embedding.

Let $A \in \mathcal{A}$ and let $\sigma \subset A$ be an $n$-simplex. Fix an isometry $\alpha_{0}$ of $\sigma$ with the fundamental chamber $\sigma_{0}$ of a Coxeter complex $\Sigma_{W}$. This isometry extends uniquely to an isometry $\alpha: A \rightarrow \Sigma_{W}$. Since every $n$-simplex in $A$ is isometric to $\sigma_{0}, \alpha$ is also a simplicial isomorphism. Thus, $A$ is a Coxeter complex. Moreover, if $A^{\prime} \in \mathcal{A}$ also contains $\sigma$, and $\alpha^{\prime}: A^{\prime} \rightarrow \Sigma_{W}$ is an isometry extending $\alpha_{0}$, then $\alpha^{-1} \circ \alpha^{\prime}: A \rightarrow A^{\prime}$ is an isometry fixing $\sigma$ and hence fixing all of $A \cap A^{\prime}$. Finally, since $\mathcal{A}$ contains a system of apartments, any two simplices of $X$ are contained in some $A \in \mathcal{A}$. Thus, $\mathcal{A}$ satisfies the conditions for a system of apartments.

\section{Spherical buildings}

In this section we prove a partial converse to Proposition 2.3. It will form the inductive step to one of the main theorems in Section 7.

Theorem 3.1 Suppose $X$ is a connected, piecewise spherical cell complex of dimension $n \geq 2$ satisfying

(1) $X$ is $C A T(1)$,

(2) $l k(x, X)$ is a spherical building for every vertex $x \in X$.

Then $X$ is a spherical building.

Before embarking on the proof, we make several observations about the hypotheses. First, If $\sigma$ is any cell in $X$, then $L_{\sigma}=\operatorname{lk}(\sigma, X)$ is a spherical building. For if $v$ is any vertex of $\sigma$, then

$$
L_{\sigma}=\operatorname{lk}(\mathrm{lk}(v, \sigma), \operatorname{lk}(v, X))
$$

and $\mathrm{lk}(v, X)$ is a spherical building by hypothesis. Thus, it follows from Proposition $2.3(2)$ that $L_{\sigma}$ is a spherical building. Moreover, if $x$ is any point in $X$, 
not necessarily a vertex, then $\mathrm{lk}(x, X)$ is also a spherical building. For if $x$ lies in the relative interior of a $k$-cell $\sigma$, then $\mathrm{lk}(x, X)=\mathbb{S}^{k-1} * L_{\sigma}=\Sigma^{k}\left(L_{\sigma}\right)$.

By Proposition 2.3, there is an obvious candidate for a system of apartments for $X$, namely

$$
\mathcal{A}=\left\{A \subset X \mid A \text { is isometric to } \mathbb{S}^{n}\right\} .
$$

As in the proof of Proposition 2.3(3), it is easy to show that any such subspace $A$ is a subcomplex of $X$ and the inclusion $A \hookrightarrow X$ is an isometric embedding.

The key problem in the proof of Theorem 3.1 is to construct enough of these subcomplexes. The idea is as follows. For any pair of antipodal points (two points are antipodal if they have distance $\pi)$ and any apartment $A_{x}$ in $\operatorname{lk}(x, X)$, we construct an apartment $A$ in $X$ by propagating geodesics from $x$ to $y$ in every direction in $A_{x}$.

We begin with a key technical lemma. Some additional notation will be needed for the proof. If $x \in X$ and $\gamma$ is a geodesic emanating from $x$, let $\gamma_{x} \in \operatorname{lk}(x, X)$ denote the tangent vector to $\gamma$ at $x$. Let st $(x)$ denote the closed star of $x$, that is, st $(x)$ is the union of the closed simplices containing $x$. (In the locally spherical context, st $(x)$ will denote a conelike polyhedral neighborhood of $x$.)

For $\sigma$ a spherical $(n-1)$-cell, the spherical suspension $\Sigma(\sigma)$, viewed as a subspace of $\mathbb{S}^{n}$, is called a spherical sector. When $n=2$, it is also called a spherical lune.

We prove the next lemma under slightly more general hypotheses for use in the next section. In particular, we do not assume that $X$ is globally CAT(1).

Lemma 3.2 Suppose $X$ is a locally spherical space of dimension $n \geq 2$ such that the link of every point in $X$ is isometric to a building. Let $\gamma$ be a local geodesic of length $\pi$ from $x$ to $y$ and let $A_{x}$ be an apartment in $L_{x}$ containing $\gamma_{x}$. Then there is a neighborhood $N_{x} \subset A_{x}$ of $\gamma_{x}$ and a unique locally isometric map $F$ of the spherical sector $\Sigma\left(N_{x}\right)$ into $X$ such that

(1) for any $v \in N_{x}$, the restriction of $F$ to $\Sigma(v)\left(=\mathbb{S}^{0} *\{v\}\right)$ is a local geodesic from $x$ to $y$ with tangent vector $v$, and

(2) the restriction of $F$ to $\Sigma\left(\gamma_{x}\right)$ is precisely $\gamma$.

Proof Divide $\gamma$ into segments $\gamma_{1}, \gamma_{2}, \ldots, \gamma_{k}$ with endpoints $x=x_{0}, x_{1}, \ldots, x_{k}$ such that each $\gamma_{i}$ lies in st $\left(x_{i-1}\right)$. Let $N_{x}$ be an $\epsilon$-ball in $A_{x}$ centered at $\gamma_{x}$ and let $S$ be the spherical sector $S=\Sigma\left(N_{x}\right)$.

For each vector $v \in N_{x}$, there is a unique geodesic segment $\gamma_{1}^{v}$ in st $(x)$ from $x$ to $\partial($ st $(x))$ whose tangent at $x$ is $v$. Let $B^{1}$ be the subspace of st $(x)$ consisting 
of the union of these geodesic segments. Identifying $\gamma_{1}^{v}$ with an initial segment of $\Sigma(v)$ gives an isometry $F_{1}$ of a polyhedral subspace of $S$ onto $B_{1}$.

Next, consider the $(n-1)$-dimensional building $L_{1}=\operatorname{lk}\left(x_{1}, X\right)$. The tangent vectors to $\gamma_{1}$ and $\gamma_{2}$ at $x_{1}$ form a pair of antipodal points $a_{1}, a_{2}$ in $L_{1}$ (since the concatenation $\gamma_{1} \cdot \gamma_{2}$ is geodesic), and $\mathrm{lk}\left(x_{1}, B_{1}\right)$ is a neighborhood of $a_{1}$ in $L_{1}$ isometric to a spherical $(n-1)$-cell. The union of geodesics in $L_{1}$ from $a_{1}$ to $a_{2}$ with an initial segment lying in this spherical cell forms an apartment $A_{1} \subset L_{1}$. The geodesic segments emanating from $x_{1}$ in directions $A_{1}$ form a spherical $n$-cell $C$ in st $\left(x_{1}\right)$. Shrinking the original $\epsilon$-neighborhood $N_{x}$ if necessary, we may assume that all of the segments $\gamma_{1}^{v}$ end in $C$. There is then a unique locally geodesic continuation of $\gamma_{1}^{v}$ across $C$, ending in $\partial\left(\operatorname{st}\left(x_{1}\right)\right)$. Call this new segment $\gamma_{2}^{v}$. Let $B_{2}$ be the union of the local geodesics $\gamma_{1}^{v} \cdot \gamma_{2}^{v}, v \in N_{x}$. Then $F_{1}$ extends in an obvious manner to a local isometry $F_{2}$ from a polyhedral subspace of $S$ onto $B_{2}$.

We repeat this process at each $x_{i}$ until we get geodesics $\gamma^{v}=\gamma_{1}^{v} \cdot \gamma_{2}^{v} \cdots \gamma_{k}^{v}$ for every $v \in N_{x}$ and a local isometry $F$ from $S$ onto $B_{k}=\bigcup \gamma^{v}$ as required.

Returning to the hypothesis of Theorem 3.1, we can now construct apartments in $X$.

Lemma 3.3 Let $X$ be as in Theorem 3.1. Suppose $x, y \in X$ are antipodal points and $A_{x}$ is an apartment in $L_{x}=l k(x, X)$. Then the following hold.

(1) For every $v \in A_{x}$, there exists a unique geodesic $\gamma^{v}$ from $x$ to $y$ whose tangent vector at $x$ is $v$.

(2) The union of all such $\gamma^{v}, v \in A_{x}$, is isometric to $\mathbb{S}^{n}$.

Proof First note that since $X$ is $\operatorname{CAT}(1)$, any local geodesic of length $\leq \pi$ is a geodesic. Moreover, if two geodesics from $x$ to $y, \gamma$ and $\gamma^{\prime}$, have the same tangent vectors $\gamma_{x}=\gamma_{x}^{\prime}=v$, then they must agree inside st $(x)$, hence they must agree everywhere. (Otherwise, we get a geodesic digon of length $<2 \pi$.) Thus, geodesics from $x$ to $y$ are uniquely determined by their tangents at $x$.

Consider the set $C=\left\{v \in A_{x} \mid \gamma^{v}\right.$ exists $\}$. By Lemma 3.2, this set is open in $A_{x}$. We claim that it is also closed. To see this, first note that if $v_{1}, v_{2} \in C$ are points of distance $\alpha$ in $A_{x}$, then for any $t \in[0, \pi], d\left(\gamma^{v_{1}}(t), \gamma^{v_{2}}(t)\right) \leq \alpha$. This can be seen by comparing the digon formed by $\gamma^{v_{1}}, \gamma^{v_{2}}$ in $X$ with a digon $\gamma_{1}^{\prime}, \gamma_{2}^{\prime}$ of angle $\alpha$ connecting a pair of antipodal points $x^{\prime}, y^{\prime}$ in $\mathbb{S}^{2}$. Inside st $(x)$, these two digons are isometric. That is, for sufficiently small $\epsilon$, the distance from $z_{1}=\gamma^{v_{1}}(\epsilon)$ to $z_{2}=\gamma^{v_{2}}(\epsilon)$ in $X$ is the same as the distance between 
the corresponding points $z_{1}^{\prime}, z_{2}^{\prime}$ in $\mathbb{S}^{2}$. Thus, $z_{1}^{\prime}, z_{2}^{\prime}, y^{\prime}$ is a spherical comparison triangle for $z_{1}, z_{2}, y$. It follows from the CAT(1) condition that the distance between $\gamma^{v_{1}}(t)$ and $\gamma^{v_{2}}(t)$ in $X$ is less than or equal to the corresponding distance in $\mathbb{S}^{2}$ for all $t$. In particular, if $\left(v_{i}\right)$ is a sequence of points in $A_{x}$ converging to $v$ with $v_{i} \in C$, then $\left(\gamma^{v_{i}}\right)$ converges uniformly to a path $\gamma$ from $x$ to $y$ with $\gamma_{x}=v$. This path has length $\pi$ since each $\gamma^{v_{i}}$ has length $\pi$. Hence $\gamma$ is geodesic and $v \in C$.

Since $C$ is both open and closed, it is either empty or all of $A_{x}$. Since $X$ is a geodesic metric space, there must exist at least one geodesic $\gamma$ from $x$ to $y$. If $v$ is any point in $L_{x}$, then there exists an apartment $A_{x}$ containing both $\gamma_{x}$ and $v$. For this apartment, $C$ is nonempty, hence $v \in C$. Thus, there is a geodesic $\gamma^{v}$ with tangent vector $v$ as desired. This proves (1).

By Lemma 3.2, we know that the map $F: \Sigma\left(A_{x}\right) \rightarrow X$ taking $\Sigma(v)$ to $\gamma^{v}$ is locally isometric. Since local geodesics of length $\leq \pi$ are geodesic in $X$, this map is an isometry onto its image. This proves (2).

The spheres constructed in Lemma 3.3 give us a large number of apartments. It is now easy to show that $X$ is a building.

Lemma 3.4 $X$ has diameter $\pi$.

Proof Since every point in $\mathrm{lk}(x, X)$ has an antipodal point, geodesics in $X$ are locally extendible. Since $X$ is CAT(1), any local geodesic of length $\pi$ is a geodesic. Thus, $\operatorname{diam}(X) \geq \pi$. Suppose there exists a geodesic $\gamma:[0, d] \rightarrow X$ with $d>\pi$. Let $y=\gamma(0)$ and let $x=\gamma(\pi)$. Let $v \in \mathrm{lk}(x, X)$ be the outgoing tangent vector to $\gamma$ (ie, the tangent vector to $\left.\gamma\right|_{[\pi, d]}$ ). By Lemma 3.3, there is a geodesic $\alpha$ from $x$ to $y$ with tangent vector equal to $v$. But this means that $\alpha$ and $\left.\gamma\right|_{[\pi, d]}$ agree in a neighborhood of $x$. This is clearly impossible since the distance from $y$ decreases along $\alpha$ and increases along $\left.\gamma\right|_{[\pi, d]}$.

Lemma 3.5 If $A \in \mathcal{A}$, then $A$ is a Coxeter complex $\Sigma_{W}$ or a suspension $\mathbb{S}^{k} * \Sigma_{W}$.

Proof Let $A \in \mathcal{A}$. We first show that the $(n-1)$-skeleton of $A, A^{n-1}$, is a union of geodesic $(n-1)$-spheres which is closed under reflection across each such sphere.

Suppose $x$ is a point in the relative interior of a $(k-1)$-simplex $\sigma \subset A^{n-1}$. Then $\operatorname{lk}(\sigma, A)$ is isometric to $\mathbb{S}^{n-k}$, hence it is an apartment in the $(n-k)$ dimensional building $\mathrm{lk}(\sigma, X)$. The $(n-k-1)$-skeleton of this apartment 
is a union of geodesic $(n-k-1)$-spheres closed under reflection. Taking the join with $\mathbb{S}^{k-2}=\mathrm{lk}(x, \sigma)$, we see that $\mathrm{lk}\left(x, A^{n-1}\right)$ is a union of geodesic $(n-2)$-spheres in $\mathrm{lk}(x, A)$ closed under reflections. It follows that, in a conelike neighborhood of $x$ in $A$, the $(n-1)$-skeleton consists of a union of geodesic $(n-1)$-disks, $D_{1}, D_{2}, \ldots, D_{k}$. In particular, any geodesic through $x$ which enters this neighborhood through $A^{n-1}$ must also leave through $A^{n-1}$. Since this is true at every point $x \in A$, we conclude that any geodesic in $A$ containing a non-trivial segment in $A^{n-1}$, lies entirely in $A^{n-1}$.

Now let $D_{i}$ be one of the geodesic disks at $x$ as above. The geodesic segments in $D_{i}$ emanating from $x$ extend to form a geodesic $(n-1)$-sphere $H_{i}$ in $A$ which, by the discussion above, lies entirely in $A^{n-1}$. We call $H_{i}$ a "wall" through $x$. Reflection of $A$ across $H_{i}$ fixes $x$ and permutes the disks $D_{1}, \ldots, D_{k}$, hence it permutes the walls through $x$. Moreover, if $H^{\prime}$ is a wall through some other point $x^{\prime} \in A^{n-1}$, then $H^{\prime}$ must intersect $H_{i}$ (since they are two geodesic $(n-1)$-spheres in a $n$-sphere). Say $z \in H_{i} \cap H^{\prime}$. Applying the argument above with $x$ replaced by $z$ shows that reflection across $H_{i}$ takes $H^{\prime}$ to some other wall through $z$. Thus, it preserves $A^{n-1}$.

Let $W$ be the group generated by reflection across the walls of $A$. By the previous lemma, $W$ acts on $A$ as a group of simplicial isomorphisms. Since $A$ is a finite complex, $W$ is a finite reflection group, or in other words, a spherical Coxeter group. Let $A^{W}$ be the fixed set of $W$ which consists of the intersection of all the walls. Then $A^{W}$ is a geodesic $k$-sphere for some $k$, and $A$ decomposes as a join, $A=A^{W} * \Sigma_{W}$.

It follows from Lemma 3.5, that cells in $X$ are simplices or suspensions of simplices.

Lemma 3.6 Any two cells $\sigma_{1}, \sigma_{2}$ in $X$ are contained in some $A \in \mathcal{A}$.

Proof Since any cell is contained in an $n$-cell, it suffices to prove the lemma for two $n$-cells. Let $x_{1}, x_{2}$ be points in the interior of $\sigma_{1}, \sigma_{2}$, respectively. Let $\gamma$ be a geodesic from $x_{1}$ to $x_{2}$ and continue $\gamma$ to a geodesic of length $\pi$. Let $y$ be the endpoint of $\gamma$ antipodal to $x_{1}$ and note that $\mathrm{lk}\left(x_{1}, X\right)=\mathrm{lk}\left(x_{1}, \sigma_{1}\right) \cong \mathbb{S}^{n-1}$. It follows from Lemma 3.3 that the union of all geodesics from $x_{1}$ to $y$ forms a subspace $A \in \mathcal{A}$. Since $A$ is a subcomplex and contains both $x_{1}$ and $x_{2}$, it must contain $\sigma_{1}$ and $\sigma_{2}$.

Lemma 3.7 If $A_{1}, A_{2} \in \mathcal{A}$ share a common chamber $\sigma$, then there is a simplicial isomorphism $\phi: A_{1} \rightarrow A_{2}$ fixing $A_{1} \cap A_{2}$ pointwise. Moreover, $A_{1}^{W}=$ $A_{2}^{W}$. 
Proof Let $x$ be a point in the relative interior of $\sigma$. Let $p$ denote the north pole of $\mathbb{S}^{n}$ and fix an isometry $\theta: \operatorname{lk}(x, \sigma) \rightarrow \mathbb{S}^{n-1}=\mathrm{lk}\left(p, \mathbb{S}^{n}\right)$. Then there is a unique isometry $\phi_{i}: A_{i} \rightarrow \mathbb{S}^{n}$ with $\phi_{i}(x)=p$ and the induced map on $\mathrm{lk}\left(x, A_{i}\right)=\mathrm{lk}(x, \sigma)$ equal to $\theta$. Let $\phi=\phi_{2}^{-1} \circ \phi_{1}$. Since the cell structure of $A_{i}$ is completely determined by reflection in the walls of $\sigma$, the isometry $\phi$ is also a simplicial isomorphism. For any point $y \in A_{1} \cap A_{2}$ not antipodal to $x$, there is a unique geodesic $\gamma$ from $x$ to $y$ which necessarily lies in $A_{1} \cap A_{2}$. Since $\phi_{1}$ and $\phi_{2}$ agree on the tangent vector $\gamma_{x}$, they agree on all of $\gamma$.

The last statement of the lemma follows from the fact that $A_{i}^{W}=\sigma^{W}$.

It follows from Lemma 3.7, that $X$ itself decomposes as a join of $A^{W}$ and a spherical building with Coxeter group $W$. This completes the proof of Theorem 3.1 .

If we are not given an a priori cell structure, we can work in the setting of locally spherical spaces and use the singular set (ie, the branch set) of $X$ to define a cell structure. In this setting we get the following theorem.

Theorem 3.8 Suppose $X$ is a locally spherical space of dimension $n \geq 2$ satisfying

(1) $X$ is $C A T(1)$,

(2) $l k(x, X)$ is isometric to a building for every point $x \in X$.

Then $X$ is isometric to a spherical building. The cell structure determined by the singular set is that of a thick, spherical building or a suspension of a thick, spherical building.

\section{More on spherical buildings}

In contrast to the locally Euclidean case, a locally spherical space which is simply connected and locally CAT(1) need not be globally CAT(1). However, as we now show, under the stronger hypothesis that links are isometric to buildings, a simply connected locally spherical space of dimension $\geq 3$ is $\operatorname{CAT}(1)$, and hence is also isometric to a spherical building.

Theorem 4.1 Suppose $X$ is a locally spherical space of dimension $n \geq 3$ satisfying

(1) $X$ is simply connected,

(2) $l k(x, X)$ is isometric to a building for every point $x \in X$.

Then $X$ is CAT(1), hence it is isometric to a building. 
The hypothesis that $n \geq 3$ is essential here. In the 1980's there was much interest in the the relation between incidence geometries and buildings (see for example [14] and [10]). In [14], Tits proves a theorem analogous to Theorem 4.1 for incidence geometries, with the same dimension hypothesis. A counterexample in dimension $n=2$ is given by Neumaier in [12]. It is a finite incidence geometry of type $C_{3}$, with a transitive action of $A_{7}$ (the alternating group on 7 letters). The flag complex associated to Neumaier's $A_{7}$-incidence geometry is a 2-dimensional simplicial complex, all of whose links are buildings, but which cannot be covered by a building. One can metrize Neumaier's example by assigning every 2 -simplex the metric of a spherical triangle with angles $\frac{\pi}{2}, \frac{\pi}{3}, \frac{\pi}{4}$. Passing to the universal cover gives a counterexample to Theorem 4.1 in dimension $n=2$.

Before proving the theorem, we will need some preliminary lemmas. We begin with an easy consequence of Lemma 3.2.

Lemma 4.2 Let $X$ be as in Theorem 4.1 and let $\gamma$ be a local geodesic in $X$ of length $\pi$ from $x$ to $y$. Then there is a unique locally isometric extension $F_{\gamma}: \Sigma\left(L_{x}\right) \rightarrow X$ of $\gamma$ (in the sense of Lemma 3.2, (1) and (2)).

Proof Suppose $A$ is an apartment in $L_{x}$ containing the tangent vector $v=\gamma_{x}$. Then by Lemma 3.2, there is a neighborhood $U$ of $v$ in $A$ and a unique locally isometric map $F_{U}: \Sigma(U) \rightarrow X$ whose restriction to $\Sigma(v)$ is $\gamma$. Using the maps $F_{U}$, we can extend $\gamma$ uniquely along any geodesic in $L_{x}$ beginning at $v$. Since $L_{x}$ is simply connected for $n \geq 3$, these extensions are compatible.

Suppose $\gamma_{1}$ and $\gamma_{2}$ are two local geodesics of length $\pi$ from $x$ to $y$ and let $v_{i}=\left(\gamma_{i}\right)_{x}$. Then it follows from the construction of $F_{\gamma_{i}}$ that the following are equivalent.

(1) $F_{\gamma_{1}}=F_{\gamma_{2}}$.

(2) $\gamma_{2}$ is the restriction of $F_{\gamma_{1}}$ to $\Sigma\left(v_{2}\right)$ (and vice versa).

(3) There exists a locally isometric map of a spherical lune into $X$ with sides $\gamma_{1}$ and $\gamma_{2}$.

We say that a geodesic $\eta:[0, a] \rightarrow X$ from $x$ is nonbranching if any other geodesic $\eta^{\prime}:[0, a] \rightarrow X$ from $x$ with $\eta_{x}=\eta_{x}^{\prime}$ is equal to $\eta$. (Or in other words, $\eta$ has unique continuation at every point in its interior.) In particular, if $\eta$ is contained in a cone-like neighborhood of $x$, then it is non-branching.

The following is an immediate consequence of Lemma 4.2. 
Lemma 4.3 Suppose $\gamma$ and $\eta$ are geodesics of length $\leq \pi$ starting at $x$ and assume $\eta$ is nonbranching. Then there is a locally isometric map of a spherical triangle into $X$ (possibly a geodesic or a spherical lune) which restricts on two sides to $\gamma$ and $\eta$.

The local isometry in the corollary above is essentially unique. More precisely, we have the following.

Lemma 4.4 Let $T_{1}$ and $T_{2}$ be spherical triangles and $\Theta_{1}: T_{1} \rightarrow X$ and $\Theta_{2}: T_{2} \rightarrow X$ be local isometries. If $\Theta_{1}$ and $\Theta_{2}$ agree along two edges of the triangle, then one of the following holds.

(1) $T_{1}=T_{2}$ (ie, they are isometric) and $\Theta_{1}=\Theta_{2}$,

(2) $T_{1}$ and $T_{2}$ are hemispheres and $\Theta_{1}, \Theta_{2}$ agree along their entire boundary.

(3) $T_{1}$ and $T_{2}$ are spherical lunes and the two edges along which they agree form one entire side of the lune.

Proof By hypothesis, $\Theta_{1}$ and $\Theta_{2}$ restrict along two edges to local geodesics $\gamma$ and $\eta$ emanating from some point $x$. The angle between these two edges is the distance in $L_{x}$ between $\gamma_{x}$ and $\eta_{x}$. Suppose this angle less than $\pi$. Then clearly $T_{1}=T_{2}$. Since $X$ is locally $\mathrm{CAT}(1)$, the subspace of $T_{1}$ on which $\Theta_{1}=\Theta_{2}$ must be locally convex, and hence must be all of $T_{1}$.

If the angle between the edges is exactly $\pi$, then $T_{i}$ is either a geodesic (if length $(\gamma)+$ length $(\eta)<\pi$ ), a spherical lune (if length $(\gamma)+$ length $(\eta)=\pi$ ), or a hemisphere (if length $(\gamma)+$ length $(\eta)>\pi$ ). In the last case, we may assume without loss of generality that length $(\gamma)=\pi$. then $\Theta_{1}$ and $\Theta_{2}$ are both restrictions of $F_{\gamma}: \Sigma L_{x} \rightarrow X$. In particular, they agree along $\left(\Sigma \gamma_{x}\right) \cup\left(\Sigma \eta_{x}\right)$ which forms the boundary of $T_{i}$.

Lemma 4.5 The diameter of $X$ is at most $\pi$.

Proof The proof is the same as that of Lemma 3.4 (using Lemma 4.2 in place of Lemma 3.3). 
Proof of Theorem 4.1 Fix a point $x$ in $X$. Define an equivalence relation on the set of geodesics of length $\pi$ starting at $x$ by

$$
\gamma_{1} \sim \gamma_{2} \Longleftrightarrow F_{\gamma_{1}}=F_{\gamma_{2}}
$$

To prove Theorem 4.1, we define a covering space $f: \tilde{X} \rightarrow X$ as follows. As a set, $\tilde{X}$ is defined as the quotient

$$
\tilde{X}=\{\gamma \mid \gamma \text { is a local geodesic of length } \leq \pi \text { with } \gamma(0)=x\} / \sim
$$

Note that only local geodesics of length $\pi$ can be identified in $\tilde{X}$.

The topology on $\tilde{X}$ is defined as follows. Let $B_{r}(y)$ denote the ball of radius $r$ in $X$ centered at $y$. Given a local geodesic $\gamma$ from $x$ to $y$ and a real number $r$ such that $B_{r}(y)$ is conelike, define

$$
\begin{gathered}
B_{r}(\gamma)=\{\eta \in \tilde{X} \mid \exists \text { a locally isometric map of a spherical triangle } \\
\text { into } X \text { which restricts on two sides to } \gamma \text { and } \eta \\
\text { and whose third side lies in } \left.B_{r}(y)\right\} .
\end{gathered}
$$

If $\gamma$ has length $\pi$, then these locally isometric maps are all restrictions of $F_{\gamma}$. In particular, $B_{r}(\gamma)$ depends only on the class of $\gamma$ in $\tilde{X}$. These sets form a basis for the topology on $\tilde{X}$. They also define a metric (locally) on $\tilde{X}$. Namely, the distance between $\gamma$ and $\eta \in B_{r}(\gamma)$ is the length of the third side of the triangle.

Define $f: \tilde{X} \rightarrow X$ to be the map taking $\gamma$ to its endpoint. By Lemma 4.3, $f$ restricts to an isometry of $B_{r}(\gamma)$ onto $B_{r}(y)$. Letting $\gamma$ run over all local geodesics from $x$ to $y$, we claim that these balls make up the entire inverse image of $B_{r}(y)$. For suppose $\eta \in \tilde{X}$ with $f(\eta)=z \in B_{r}(y)$. Since $z$ lies in a conelike neighborhood of $y$, the geodesic $\delta$ from $z$ to $y$ is nonbranching. It follows from Lemma 4.3, applied to $\eta$ and $\delta$, that there exists a local isometry of a spherical triangle into $X$ which restricts on two side to $\eta$ and $\delta$. The restriction to the third side, $\gamma$, is a local geodesic from $x$ to $y$ such that $\eta \in B_{r}(\gamma)$.

It remains to show that for distinct $\gamma_{i} \in \tilde{X}$, the balls $B_{r}\left(\gamma_{i}\right)$ are disjoint. Suppose $\delta \in B_{r}\left(\gamma_{1}\right) \cap B_{r}\left(\gamma_{2}\right)$. Then there is a local isometry $\Theta_{i}$ of a spherical triangle with sides $\gamma_{i}$ and $\delta$, for $i=1,2$. Since $B_{r}(y)$ is conelike, there is a unique geodesic $\eta$ from $y$ to the endpoint $z$ of $\delta$. Thus $\Theta_{1}$ and $\Theta_{2}$ agree along two edges, $\delta$ and $\eta$. It follows from Lemma 4.4 that $\gamma_{1}=\gamma_{2}$ in $\tilde{X}$.

This proves that $f: \tilde{X} \rightarrow X$ is a covering map. By hypothesis, $X$ is simply connected, and it is easy to verify that $\tilde{X}$ is connected, thus $f$ is injective. In particular, for any $y \in X$ of distance less than $\pi$ from $x$, there is a unique local geodesic from $x$ to $y$. Moreover, it follows from Lemma 4.3 that this geodesic varies continuously with the endpoint $y$. Since $x$ was chosen arbitrarily, this applies to all $x$ and $y$. By Theorem 2.2(2), we conclude that $X$ is CAT(1). 


\section{$5 \quad$ Euclidean buildings}

Theorem 5.1 Suppose $X$ is a connected, locally Euclidean complex satisfying

(1) $X$ is $C A T(0)$,

(2) for every point $x \in X, L_{x}=l k(x, X)$ is isometric to a spherical building.

Then $X$ decomposes as an orthogonal product $X=\mathbb{R}^{l} \times X_{1} \times \cdots \times X_{k}$, where $l \geq 0$, and each $X_{i}$ is one of the following,

(1) a thick, irreducible Euclidean building,

(2) the Euclidean cone on a thick, irreducible spherical building,

(3) a tree.

Remark The reader may object that a tree is a 1-dimensional irreducible Euclidean building whose apartments are Coxeter complexes for the infinite dihedral group. However, the standard building metric on a 1-dimensional Euclidean building would assign the same length to every edge of the tree. Since this need not be the case in our situation, we list these factors separately.

In [11], Kleiner and Leeb introduce a more general notion of a Euclidean building, which we will call a "metric Euclidean building", and prove an analogous product decomposition theorem (Prop. 4.9.2) for these buildings. We review their definition in the context of locally Euclidean spaces. (Kleiner and Leeb work in a more general setting.)

Call a group $W$ of affine transformations of $\mathbb{R}^{n}$ an affine Weyl group if $W$ is generated by reflections and the induced group of isometries on the sphere at infinity is finite. Affine Weyl groups include Euclidean and spherical Coxeter groups, as well as nondiscrete groups generated by reflections across parallel walls.

Let $\mathcal{A}$ be a collection of isometric embeddings of $\mathbb{R}^{n}$ into a locally Euclidean space $X$ of dimension $n$. We call $\mathcal{A}$ an atlas for $X$ and the images of the embeddings are called apartments.

Definition 5.2 Suppose $X$ is a CAT(0), locally Euclidean space of dimension $n$. Then $X$ is a metric Euclidean Building if there is an atlas $\mathcal{A}$ and an affine Weyl group $W$ such that

(1) Every geodesic segment, ray, and line is contained in an apartment.

(2) $\mathcal{A}$ is closed under precomposition with $W$.

(3) If two apartments $\phi_{2}\left(\mathbb{R}^{n}\right), \phi_{2}\left(\mathbb{R}^{n}\right)$ intersect, then $\phi_{1}^{-1} \circ \phi_{2}$ is the restriction of some element of $W$. 
(In the context of locally Euclidean spaces, this definition agrees with that of Kleiner and Leeb since their first two axioms hold automatically for locally Euclidean spaces.) It is an immediate consequence of Theorem 5.1 that the space $X$ is a metric Euclidean building.

Corollary 5.3 Let $X$ be as in Theorem 5.1. Then $X$ is a metric Euclidean building.

Conversely, it is easy to see that a metric Euclidean building satisfies the hypotheses of Theorem 5.1. Thus, for locally Euclidean spaces, Theorem 5.1 also provides another proof of Kleiner and Leeb's product decomposition theorem.

The proof of Theorem 5.1 will occupy the remainder of this section. As in the spherical case, the key is to find lots of apartments. By Proposition 2.3, the apartments in $X$ are isometrically embedded copies of $\mathbb{R}^{n}$, known as $n$ flats. The crucial step to constructing $n$-flats is to find flat strips (isometrically embedded copies of $\mathbb{R} \times[0, a]$ for some $a>0$ ).

Definition 5.4 Let $X$ be a $C A T(0)$-space. We will call the triangle $\Delta$ in $X$ a Euclidean (or flat) triangle, if its convex hull is isometric to a triangle in $\mathbb{R}^{n}$.

For a triangle $\Delta(x, y, z)$ we denote the segment from $x$ to $y$ by $x y$, etc. The angle between $x y$ and $x z$ is defined as the distance in $L_{x}$ between the tangent vectors to $x y$ and $x z$ and it is denoted by $\angle_{x}(x y, x z)$. The following lemma follows immediately from Proposition 3.13 of [1].

Lemma 5.5 Let $X$ be a $C A T(0)$ space, $\Delta=\Delta(a, b, c)$ a triangle in $X$. Let $d$ be a point between $a$ and $b$. Suppose the triangles $\Delta(a, d, c)$ and $\Delta(b, d, c)$ are Euclidean. If in addition $\angle_{d}(d a, d c)+\angle_{d}(d b, d c)=\pi$, then the original triangle $\Delta(a, b, c)$ is Euclidean.

The condition on the angles is automatically satisfied if the geodesic $a b$ is nonbranching, for example if $b$ lies in a cone-like neighborhood of $a$.

If $L$ is a locally spherical space and $r>0$, let $C_{r}(L)$ denote the Euclidean cone on $L$ of radius $r$ (ie, the geodesics emanating from the cone point have length $r$ ). The following lemma is an analogue of Lemma 3.2. The proof is essentially the same and the details are left to the reader. 
Lemma 5.6 Suppose $X$ is a locally Euclidean space of dimension $n \geq 2$ such that the link of every point in $X$ is isometric to a spherical building. Let $\gamma$ be a locally geodesic ray from $x$ and let $A_{x}$ be an apartment in $L_{x}$ containing $\gamma_{x}$. Then for any $r>0$, there is a neighborhood $N_{x} \subset A_{x}$ of $\gamma_{x}$ and a unique locally isometric map $\Theta$ of the Euclidean cone $C_{r}\left(N_{x}\right)$ into $X$ such that

(1) for any $v \in N_{x}$, the restriction of $\Theta$ to $C_{r}(v)$ is a local geodesic with tangent vector $v$, and

(2) the restriction of $\Theta$ to $C_{r}\left(\gamma_{x}\right)$ is precisely $\left.\gamma\right|_{[0, r]}$.

Suppose $\gamma$ is as in Lemma 5.6, and $\alpha$ is a geodesic in $L_{x}$ originating at $\gamma_{x}$. Then Lemma 5.6 implies that there is a unique extension of $\left.\gamma\right|_{[0, r]}$ to a locally isometric map of $C_{r}(\alpha)$ into $X$. If $X$ is $\operatorname{CAT}(0)$, the map is an isometric embedding. This enables us to construct Euclidean triangles in $X$ since the image of any triangle in $C_{r}(\alpha)$ is Euclidean.

From now on, we assume that $X$ satisfies the hypotheses of Theorem 5.1.

Lemma 5.7 Let $\gamma: \mathbb{R} \rightarrow X$ be a geodesic ray with $x=\gamma(0)$. If $y$ lies in a conelike neighborhood of $x$, then for any $t \in \mathbb{R}$, the triangle $\Delta(x, y, \gamma(t))$ is Euclidean.

Proof Assume, without loss of generality, that $t>0$. Since $y$ lies in a conelike neighborhood of $x$, the geodesic $\eta$ from $x$ to $y$ is non-branching. Choose a geodesic $\alpha$ in $L_{x}$ from $\gamma_{x}$ to $\eta_{x}$ and extend $\left.\gamma\right|_{[0, t]}$ to an isometric embedding $\Theta$ of $C_{t}(\alpha)$ into $X$. Since $\eta$ is non-branching, it agrees with $\Theta$ on $C_{t}\left(\eta_{x}\right)$. Thus, $x, y, \gamma(t)$ span a Euclidean triangle.

Lemma 5.8 Let $\gamma, x$, and $y$ be as above and let $\eta$ be the geodesic from $x$ to $y$. Suppose $\angle_{x}\left(\eta, \gamma^{+}\right)+\angle_{x}\left(\eta, \gamma^{-}\right)=\pi$. Then $y$ and $\gamma$ span a Euclidean strip.

Proof By the previous lemma, for each $t \in \mathbb{R}$, the triangle $\triangle(x, y, \gamma(t))$ is Euclidean. By Lemma 5.5, every triangle of the form $\triangle\left(y, \gamma\left(t_{1}\right), \gamma\left(t_{2}\right)\right)$ is Euclidean. Since any two points in the span of $y$ and $\gamma$ lie on such a triangle, the lemma follows.

For any subspace $Y$ of $X$, and any point $x \in Y$, we denote the link of $x$ in $Y$ by $L_{x} Y$.

Lemma 5.9 Let $F$ be an $m$-flat in $X$. Let $\eta:[0, r] \rightarrow X$ be a geodesic from $x \in F$ to a point $y$ lying in a conelike neighborhood of $x$. Suppose that the distance in $L_{x}$ from $\eta_{x}$ to any point in $L_{x} F$ is $\frac{\pi}{2}$. Then $y$ and $F$ span a flat $R^{m} \times[0, r]$. 
Proof Let $Z=\mathbb{R}^{m} \times[0, r]=F \times[0, r]$. Let $Y$ be the subspace of $X$ spanned by $F$ and $y$. Consider the natural map $f: Z \rightarrow Y$ that takes $F \times\{0\}$ via the identity map to $F$ and takes the line segment between $(z, 0)$ and $(0, r)$ to the geodesic in $Y$ from $z$ to $y$. By Lemma 5.8, the restriction of $f$ to the strip $\gamma \times[0, r]$ is an isometry for any geodesic $\gamma: \mathbb{R} \rightarrow F$ through $x$. We must show that $f$ is isometric on all of $Z$.

Any two points $y_{1}, y_{2}$ in $Y$ lie on the image of a triangle $T \subset Z$ with vertices $(0, r),\left(z_{1}, 0\right),\left(z_{2}, 0\right)$. By the discussion above, $T$ is a comparison triangle for its image $f(T)$ in $Y$. Hence, by the $\operatorname{CAT}(0)$ condition, the distance between $y_{1}$ and $y_{2}$ is at most the distance between the corresponding points in $T$. Thus, $f$ is distance non-increasing. Moreover, if $y_{1}$ or $y_{2}$ lies on $\eta$, then they both lie in a Euclidean strip, as above, so these two distances agree.

To prove the reverse inequality, choose $r^{\prime}<r$ and let $y^{\prime}=\eta\left(r^{\prime}\right)=f\left(\left(0, r^{\prime}\right)\right)$ (Figure 1). Consider the induced map $d f$ between the links $L_{\left(0, r^{\prime}\right)} Z \cong \mathbb{S}^{m}$ and $L_{y^{\prime}} Y$. It suffices to prove that $d f$ is an isometry, for in this case, the triangle with vertices $y^{\prime}, y_{1}, y_{2}$ has the same angle and same two side lengths at $y^{\prime}$ as its comparison triangle in $Z$, so by the $\mathrm{CAT}(0)$ condition, the opposite side is at least as long as in the comparison triangle.

To see that $d f$ is an isometry, note that the fact that $f$ restricts to an isometry on strips $\gamma \times[0, r]$ implies that $d f$ maps points of distance $\pi$ in $L_{\left(0, r^{\prime}\right)} Z$ to points at distance $\pi$ in $L_{y^{\prime}} Y$. On the other hand, since $f$ is distance nonincreasing at all points, it must also be distance non-increasing on links. But these two facts contradict each other unless $d f$ is an isometry.

Lemma 5.10 Every geodesic and every flat strip in $X$ is contained in an $n$-flat.

Proof Let $F$ be an $m$-flat in $X$ with $m<n$. Let $a \in[-\infty, 0], b \in[0, \infty]$ be chosen so that $F$ is contained in a flat embedded $F \times[a, b]$ (with $F=F \times\{0\}$ ) and such that $a, b$ are maximal, (that is, one cannot embed this $F \times[a, b]$ in a bigger $\left.F \times\left[a_{0}, b_{0}\right]\right)$.

We claim that $a=-\infty, b=\infty$. Assume the contrary. Say, for example, $b<\infty$. Let $x$ be a point in $F \times\{b\} . F \times[a, b]$ determines an $m$-dimensional hemisphere $H$ in $L_{x} X$ containing $L_{x} F$. (If $a=b=0$, choose any hemisphere containing $L_{x} F$.) Choose an $m$-sphere in $L_{x} X$ containing $H$ and let $v$ be the point of the sphere, which has distance $\frac{\pi}{2}$ from $H$. Let $\eta$ be a ray emanating from $x$ in the direction of $v$. By the previous lemma, for small $r, \eta(r)$ and $F \times\{b\}$ span a flat $F \times[0, r]$. The choice of $v$, together with Lemma 5.5, guarantee that $F \times[a, b]$ and $F \times[0, r]$ fit together to form a flat strip $F \times[a, b+r]$. This contradicts the maximality of $b$. 


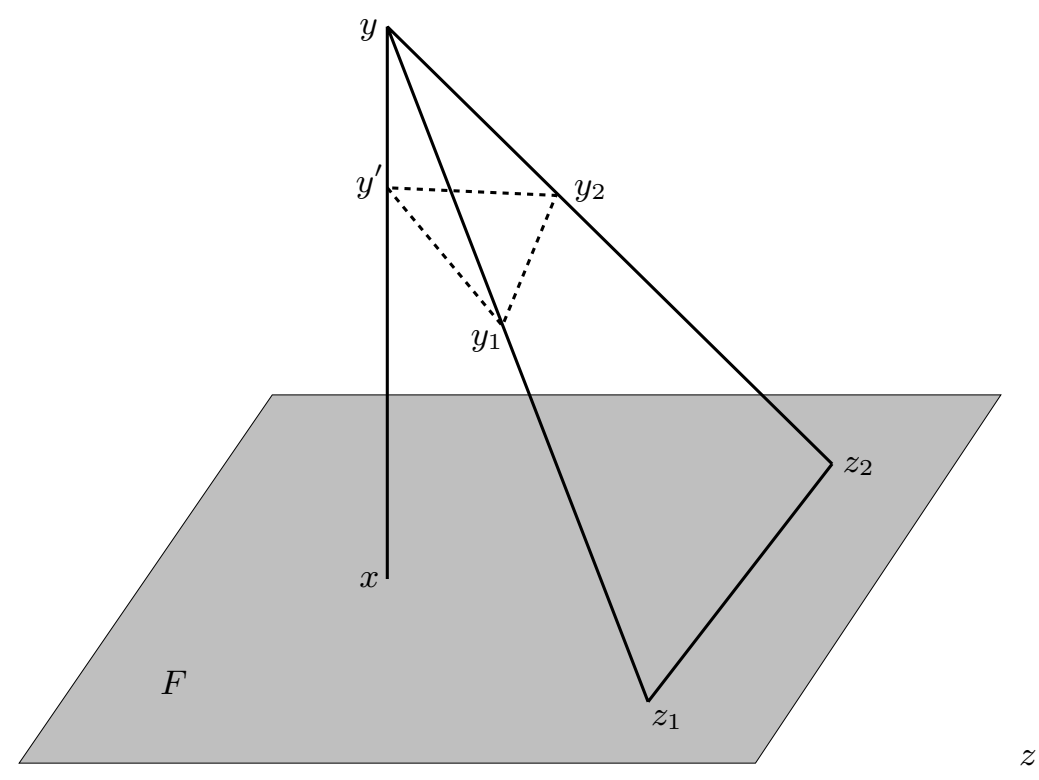

Figure 1

Thus, any $m$-flat, and more generally, any geodesically embedded $\mathbb{R}^{m} \times[a, b]$, $m<n$, can be embedded in an $(m+1)$-flat. It follows inductively, that every geodesic and every flat strip is contained in an $n$-flat.

Proof of Theorem 5.1 Let $F$ be an $n$-flat in $X$. The set $Y$ of the singular points in $F$ is closed and locally it is a union of hyperplanes, so $Y$ is globally a union of hyperplanes too. We call these the singular hyperplanes. The set of singular hyperplanes is locally invariant under reflection in each of these hyperplanes, so if two singular hyperplanes $H_{1}$ and $H_{2}$ are not parallel, the reflection, $H_{1}^{\prime}$, of $H_{1}$ across $H_{2}$ is also singular. Moreover, if $H_{1}$ and $H_{2}$ are parallel, and there exists a singular hyperplane not orthogonal to $H_{1}$ and $H_{2}$, then a simple exercise shows that $H_{1}^{\prime}$ can be obtained by a series of reflections across intersecting singular hyperplanes. Thus, again, $H_{1}^{\prime}$ must be a singular hyperplane.

Let $Y_{1}, Y_{2}, \ldots, Y_{k}$ be a maximal decomposition of $Y$ into mutually orthogonal families of singular hyperplanes. It follows from the discussion above that each $Y_{i}$ is either a discrete family of parallel hyperplanes or the set of walls of an irreducible spherical or Euclidean Coxeter group (compare [6], VI.1).

Taking $F_{i}$ to be the subspace of $F$ generated by the normal vectors to the hyperplanes in $Y_{i}$, we obtain an orthogonal decomposition of $F$ into $F=$ $F_{0} \times F_{1} \times \cdots \times F_{k}$, where $F_{0}$ is a subspace parallel to all of the singular 
hyperplanes. If $Y_{i}$ is a family of parallel hyperplanes, then $F_{i}$ is 1 -dimensional. Otherwise, $Y_{i}$ gives $F_{i}$ the structure of a Euclidean Coxeter complex or an (infinite) Euclidean cone on a spherical Coxeter complex.

We now choose an $n$-flat $E$ such that, in the decomposition $E=E_{0} \times E_{1} \times$ $\cdots \times E_{k}$, the dimension $l$ of the factor $E_{0}$ is minimal, and among all flats with $\operatorname{dim}\left(E_{0}\right)=l$, we require that $E$ minimize the number $k$. (As we will see below, the number $k$ is actually independent of the choice of $E$.) In this flat $E$, we choose a point $x$, such that for $j=1, \ldots, k$, the hyperplanes $Y_{j}$ determine a full-dimensional, irreducible, spherical Coxeter group in the sphere $L_{x} E_{j}$. Intuitively, we have chosen $E$ to be the most complicated $n$-flat and $x$ to be the most complicated point in $E$.

The product decomposition of $E$ gives rise to a decomposition of $L_{x} E$ as an orthogonal join, $L_{x} E=L_{x} E_{0} * L_{x} E_{1} * \cdots * L_{x} E_{k}$. Since $L_{x} E$ is an apartment in the spherical building $L_{x} X$, it follows that $L_{x} X$ splits as a join of $k$ irreducible buildings and an $(l-1)$-sphere, $L_{x} X=L_{0} * L_{1} * \cdots * L_{k}$.

Let $G$ be another flat through $x$. As above, we can factor $G$ as an orthogonal product $G=G_{0} \times G_{1} \times \cdots \times G_{k^{\prime}}$ Since $L_{x} G$ is also an apartment in $L_{x} X$, it must have the same simplicial structure as $L_{x} E$. Clearly this is possible only if $\operatorname{dim}\left(G_{0}\right)=\operatorname{dim}\left(E_{0}\right), k^{\prime}=k$ and (up to permutation) $L_{x} G_{j} \cong L_{x} E_{j}$ for all $j$. (Note that although we have shown that the simplicial structure of $G_{j}$ and $E_{j}$ agree in a neighborhood of $x$, we do not yet know that they agree globally.)

If $y$ is any other point in $X$, then by Lemma 5.10, there is a flat $G$ containing $x$ and $y$, so the decomposition of $G$ gives rise to a decomposition of $L_{y} X$ as a join $L_{y, 0} * L_{y, 1} * \cdots * L_{y, k}$ of spherical buildings. In this case, however, the factors need not be irreducible. (Consider, for example, the case where $y$ is a nonsingular point.)

Next, we prove that if the decomposition of $E$ is not the trivial one, we can decompose $X$ as a product. Let $X_{i}$ be the union of the geodesic rays emanating from $x$ in the direction of $L_{i}$. Then $X_{i}$ is connected and by Lemma 5.7, it is locally convex, hence it is convex. To prove that $X=X_{0} \times X_{1} \times \cdots \times X_{k}$, we apply Theorem II.9.24 of [4] which states that splittings of $X$ as a product correspond to splittings of the Tits boundary $\partial_{T} X$ as a join. The Tits boundary may be viewed as the set of rays emanating from $x$. To show that $X=$ $X_{0} \times X_{1} \times \cdots \times X_{k}$, it suffices to show that $\partial_{T} X=\partial_{T} X_{0} * \partial_{T} X_{1} * \cdots * \partial_{T} X_{k}$. For this we must verify (cf [4] Lemma II.9.25):

(1) Every ray $\gamma$ lies in the span of rays $\gamma_{0}, \gamma_{1}, \ldots, \gamma_{k}$ with $\gamma_{i} \subset X_{i}$.

(2) Rays $\gamma_{i}, \gamma_{j}$ lying in distinct factors $X_{i}, X_{j}$ have distance $\frac{\pi}{2}$ in the Tits metric. 
The first of these conditions follows from the fact that any ray $\gamma$ at $x$ is contained in an $n$-flat $G$ which, by the discussion above, decomposes as a product $G=G_{0} \times G_{1} \times \cdots \times G_{k}$ with $G_{i} \subset X_{i}$.

For the second condition, it is enough to show, that any geodesic $\gamma$ in $X_{i}$ through $x$ and any ray $\eta$ in $X_{j}$ emanating from $x(i \neq j)$ span a flat halfplane. If not, then there is a largest $t$ such that $\eta(t)$ and $\gamma$ span a flat strip. By Lemma 5.8 , we can assume that $t$ is bigger than 0 . By Lemma 5.10, we can embed this strip in an $n$-flat $T$. Let $z=\eta(t)$ and let $\gamma_{1}$ be the line in $T$ parallel to $\gamma$ through $z$. The product decomposition of $T$ gives rise to a join decomposition of the building $L_{z} X=L_{z, 0} * L_{z, 1} * \cdots * L_{z, k}$. The tangent vectors to $\gamma_{1}$ at $z$ lie in $L_{z, i}$ while the tangent vectors to $\eta$ at $z$ lie in $L_{z, j}$. It follows that the angle condition of Lemma 5.8 is satisfied, so that for small $\epsilon, \eta(\epsilon+t)$ and $\gamma_{1}$ span a flat strip. This strip fits together with the strip between $\gamma_{1}$ and $\gamma$, contradicting the maximality of $t$. This proves condition (2) and we have shown that $X$ decomposes as an orthogonal product of the $X_{i}$ 's.

It remains to identify the factors $X_{i}$. Since $X_{i}$ is convex in $X$, it is $\operatorname{CAT}(0)$, and at any point $y \in X_{i}, L_{y} X_{i}=L_{y, i}$ is a building. Thus, each factor satisfies the hypotheses of Theorem 5.1. The factor $X_{0}$ contains no singular points, for if $y \in X_{0}$ is singular, then we could find an $n$-flat $F$ through $y$ with $\operatorname{dim} F_{0}<\operatorname{dim} E_{0}$. In other words, $X_{0}=\mathbb{R}^{l}$.

Assume $i>0$. If $X_{i}$ is 1 -dimensional, then the CAT(0) condition implies that it is a tree. If $\operatorname{dim} X_{i} \geq 2$, then $X_{i}$ contains an apartment $E_{i}$ which is either an irreducible Euclidean Coxeter complex or the cone on an irreducible spherical Coxeter complex. Thus, chambers in $E_{i}$ are simplices or cones on simplices, and the simplicial structure of $E_{i}$ is completely determined by a single chamber. It follows that any other apartment in $X_{i}$ sharing a chamber with $E_{i}$ has the same simplicial structure. By Lemma 5.10, any two points (hence any two chambers) in $X_{i}$ lie in a common apartment, so any two apartments are isomorphic. If $E_{i}$ is a Euclidean Coxeter complex, then we conclude that $X_{i}$ is an irreducible Euclidean building. In the case that $E_{i}$ is the cone on a spherical Coxeter complex, it has only one vertex, namely $x$, which must be the cone point for every apartment. Thus, in this case, $X_{i}$ is the cone on the irreducible spherical building $L_{i}$. In either case, since the simplicial structure on $X_{i}$ is defined by its singular set, these buildings must be thick. This completes the proof of Theorem 5.1.

It is reasonable to ask whether an analogous theorem holds for locally hyperbolic spaces; that is, if $X$ is locally hyperbolic and CAT(-1), and every link in $X$ is isometric to a spherical building, can we conclude that $X$ is a hyperbolic building (ie, a buildings whose apartments are copies of $\mathbb{H}^{n}$ cellulated by the 
walls of a discrete, hyperbolic reflection group)? The answer is no. Although one can construct lots of embedded copies of $\mathbb{H}^{n}$ under these hypotheses, these "apartments" need not have the structure of a Coxeter complex. For example, let $\gamma_{1}, \ldots \gamma_{5}$ be five geodesics $\gamma_{i}: \mathbb{R} \rightarrow \mathbb{H}^{2}$ which form a right-angled pentagon. The reflections across these lines generate a hyperbloic Coxeter group. Now let $\gamma_{6}$ be another geodesic line intersecting $\gamma_{1}$ orthogonally and disjoint from the other $\gamma_{i}$ 's. We can choose $\gamma_{6}$ so that the three geodesics intersecting $\gamma_{1}$ generate a nondiscrete group of reflections. Then $\gamma_{1}, \ldots, \gamma_{6}$ cannot be the walls of a reflection group acting on $\mathbb{H}^{2}$. (Note that these six lines are locally, but not globally closed under reflections.) It is now possible to construct a simply connected, locally hyperbolic space $X$, all of whose links are spherical buildings (in fact we can take the link of every vertex to be the $K_{3,3}$ graph with edge lengths $\frac{\pi}{2}$ ) and such that $X$ contains an isometrically embedded $\mathbb{H}^{2}$ whose singular set consists of the six lines above. This cannot be a hyperbolic building.

\section{One-dimensional spherical buildings}

In this section we give a metric characterization of one-dimensional spherical buildings. An equivalent characterization appears as Lemma 6.1 in [3]. We include a proof here for completeness.

Theorem 6.1 Suppose $X$ is a connected, one-dimensional piecewise spherical complex satisfying

(1) $X$ is $C A T(1)$ and $\operatorname{diam}(X)=\pi$,

(2) every vertex of $X$ has valence $\geq 3$.

Then $X$ is either a thick spherical building or $X=\Sigma Y$ for some discrete set $Y$.

Remark We can also apply this theorem in cases where every vertex has valence $\geq 2$ simply by ignoring those vertices of valence 2 . In this case, however, some information about the original cell structure will be lost since vertices of valence 2 are invisible to the metric.

Assume throughout this section that $X$ satisfies the hypotheses of Theorem 6.1. In this situation, the analogue of Lemma 3.3 is easy to prove.

Lemma 6.2 Let $x, y \in X$ be antipodal points and $v \in l k(x, X)$ a tangent vector at $x$. Then there exits a unique geodesic $\gamma$ from $x$ to $y$ such that $\gamma_{x}=v$. 
Proof Choose a point $z$ in the open star of $x$ so that the geodesic $\alpha$ from $x$ to $z$ satisfies $\alpha_{x}=v$. We first observe that $z$ cannot be antipodal to $y$. For if $w$ is a point on $\alpha$ between $x$ and $z$, then the geodesic from $w$ to $y$ must pass through either $z$ or $x$. If both $z$ and $x$ are antipodal to $y$, then $d(w, y)>\pi$, contradicting $\operatorname{diam}(X)=\pi$. Thus, $d(x, z)<\pi$ and hence there is a unique geodesic $\beta$ from $z$ to $y$. This geodesic cannot pass through $x$ (since this would imply $d(z, y)>d(z, x)=\pi)$, so it must leave $z$ in the opposite direction from $\alpha$. Thus, the concatenation $\alpha \cdot \beta$ is a local geodesic from $x$ to $y$. Moreover, since $\alpha$ contains no antipodal points to $y$ (other than $x$ ), the length of $\alpha \cdot \beta$ cannot be more than $\pi$. Thus, $\gamma=\alpha \cdot \beta$ is the desired geodesic.

Lemma 6.3 Suppose $x, y \in X$ are antipodal points. If $x$ is a vertex of $X$, then so is $y$.

Proof If $x$ is a vertex of $X$, then $\mathrm{lk}(x, X)$ contains at least 3 distinct points $v_{1}, v_{2}, v_{3}$. By the previous lemma, there are geodesics $\gamma_{1}, \gamma_{2}, \gamma_{3}$ from $x$ to $y$ with $\left(\gamma_{i}\right)_{x}=v_{i}$. Any two of these geodesics intersect only at $x$ and $y$ since, otherwise, they would form a circuit of length $<2 \pi$. Thus, $\operatorname{lk}(y, X)$ has at least 3 distinct points, so $y$ is a vertex.

Lemma 6.4 All edges of $X$ have the same length, $\frac{\pi}{m}$ for some integer $m \geq 1$.

Proof Since $X$ is connected, if any two edges have different lengths, then we can find a pair of adjacent edges, $e_{1}, e_{2}$ of different lengths. Let $x$ be their common vertex and let $x_{1}, x_{2}$ be the other endpoints of $e_{1}, e_{2}$ respectively. Say $d\left(x, x_{1}\right)<d\left(x, x_{2}\right)$. Choose a point $y$ antipodal to $x$ and a third edge $e_{3}$ emanating from $x$. By Lemma 6.2, there are geodesics $\gamma_{1}, \gamma_{2}, \gamma_{3}$ from $x$ to $y$ which begin with $e_{1}, e_{2}, e_{3}$ respectively. Any two of these geodesics, $\gamma_{i}, \gamma_{j}$, form a loop of length $2 \pi$. Consider the loop $\gamma_{1} \cdot \gamma_{3}$. Let $z$ be the point on $\gamma_{3}$ antipodal $x_{1}$. By Lemma 6.3, $z$ is a vertex. Next, consider the loop $\gamma_{2} \cdot \gamma_{3}$. Let $z^{\prime}$ be the point on $\gamma_{2}$ antipodal to $z$. By Lemma 6.3, $z^{\prime}$ is a vertex. But $d\left(x, z^{\prime}\right)=d(y, z)=d\left(x, x_{1}\right)<d\left(x, x_{2}\right)$. Thus, $z^{\prime}$ lies in the interior of the edge $e_{2}$. This is a contradiction. We conclude that all edges of $X$ have the same length. Since antipodal points to vertices are also vertices, this length must be $\frac{\pi}{m}$ for some integer $m \geq 1$.

If all edges of $X$ have length $\pi$, then the distance between any two vertices of $X$ is exactly $\pi$. It follows that $X$ has only two vertices (otherwise, it would have diameter $>\pi$ ). Thus, $X$ is the suspension of the discrete set $Y$ consisting of the midpoints of the edges.

From now on, we will assume that every edge has length $\frac{\pi}{m}$ for some $m \geq 2$. In this case, every circuit of length $2 \pi$ is a Coxeter complex for the dihedral group $D_{2 m}$. We will refer to such circuits as apartments. 
Lemma 6.5 Any two simplices of $X$ are contained in a common apartment. If $A_{1}$ and $A_{2}$ are apartments containing a common edge $e$, then there is a simplicial isomorphism $\phi: A_{1} \rightarrow A_{2}$ fixing $A_{1} \cap A_{2}$ pointwise.

Proof It suffices to prove the first statement for two edges $e_{1}, e_{2}$. Choose a point $x$ in the interior of $e_{1}$. Suppose $e_{2}$ contains a point $y$ antipodal to $x$ (which, by Lemma 6.3 must lie in the interior of $e_{2}$ ). Then $\mathrm{lk}\left(x, e_{1}\right)$ consists of two points $v_{1}, v_{2}$ each of which gives rise to a geodesic $\gamma_{1}, \gamma_{2}$ from $x$ to $y$. Together, these two geodesics form an apartment containing $e_{1}$ and $e_{2}$.

Suppose, on the other hand, that no point of $e_{2}$ is antipodal to $x$. Let $\gamma_{1}$ be a geodesic from $x$ to a point $y$ in the interior of $e_{2}$. Extend $\gamma_{1}$ to a geodesic of length $\pi$. Note that this extended geodesic (which we still denote $\gamma_{1}$ ) must contain all of $e_{2}$. Let $z$ be the endpoint of $\gamma_{1}$, so $z$ is antipodal to $x$. Then there exists a geodesic $\gamma_{2}$ from $x$ to $z$ which begins along $e_{1}$ in the direction opposite to $\gamma_{1}$. The union, $\gamma_{1} \cup \gamma_{2}$ is the desired apartment.

The second statement is obvious since the CAT(1) hypothesis implies that $A_{1} \cap$ $A_{2}$ is connected.

\section{Main theorems}

Combining the results of the previous sections, we arrive at our main theorems.

Theorem 7.1 Suppose $X$ is a connected, piecewise spherical complex of dimension $n \geq 2$ satisfying the following conditions.

(1) $X$ is $C A T(1)$.

(2) Every $(n-1)$-cell is contained in at least two $n$-cells.

(3) The link of every $k$-cell, $k \leq n-2$, is connected.

(4) The link of every $(n-2)$-cell has diameter $\pi$.

Then $X$ is isometric to a spherical building. The cell structure determined by the singular set is that of a thick spherical building or a suspension of a thick spherical building.

Proof Let $L_{\sigma}=\operatorname{lk}(\sigma, X)$. By Theorem 2.2, if $X$ is $\operatorname{CAT}(1)$, then so is $L_{\sigma}$ for every cell $\sigma$. In particular, for an $(n-2)$-cell $\sigma, L_{\sigma}$ is a 1 -dimensional, piecewise spherical complex which is $\operatorname{CAT}(1)$, diameter $\pi$, and has every vertex of valence $\geq 2$. Ignoring vertices of valence 2 gives a complex satisfying Theorem 6.1. Thus, $L_{\sigma}$ is isometric to a 1-dimensional spherical building. The theorem now follows by induction from Theorem 3.8. 
In the theorem above, we could have assumed that $X$ was locally spherical instead of piecewise spherical if we interpret " $k$-cell" as meaning $k$-dimensional strata of the singular set. In fact, in ignoring vertices of valence 2 , some information about the given cell structure on $X$ may be lost. For example, any cell decomposition of the standard 2-sphere satisfies the conditions of the theorem, but need not be the cell structure of a building. To guarantee that the original cell structure is reflected in the metric, we would need to assume that $X$ is thick.

Theorem 7.2 Suppose $X$ is a connected, piecewise spherical complex of dimension $n \geq 2$ satisfying the following conditions.

(1) $X$ is $C A T(1)$.

(2) Every $(n-1)$-cell is contained in at least three $n$-cells.

(3) The link of every $k$-cell, $k \leq n-2$, is connected.

(4) The link of every $(n-2)$-cell has diameter $\pi$.

Then, with respect to its given cell structure, $X$ is a spherical building.

Proof The proof is the same using Theorem 3.1 instead of Theorem 3.8.

Analogous results hold in the piecewise Euclidean setting.

Theorem 7.3 Suppose $X$ is a connected, piecewise Euclidean complex of dimension $n \geq 2$ satisfying the following conditions.

(1) $X$ is $C A T(0)$.

(2) Every $(n-1)$-cell is contained in at least two $n$-cells.

(3) The link of every $k$-cell, $k \leq n-2$, is connected.

(4) The link of every $(n-2)$-cell has diameter $\pi$.

Then $X$ is a metric Euclidean building. If, in addition, $X$ is thick, then $X$ is a product of irreducible Euclidean buildings and trees with respect to its given cell structure.

Proof The first hypothesis implies that $\mathrm{lk}(\sigma, X)$ is CAT(1) for every $\sigma$. By Theorem 7.1, we conclude that for $v$ a vertex of $X, \mathrm{lk}(v, X)$ is isometric to a spherical building. It follows by Theorem 5.1 and Corollary 5.3 that $X$ is a metric Euclidean building and that it factors as a product of irreducible Euclidean buildings, cones on spherical buildings, trees, and a nonsingular Euclidean space. If $X$ is thick, the components of the nonsingular set of $X$ are the interiors of the $n$-cells. In particular, they are bounded. Thus, only the first and last type of factor can occur in this situation. 
Replacing the CAT(1) condition in Theorem 7.1 by a simply connectedness condition does not give a satisfying characterization because of the problem in dimension 2. It does, however, give an analogue of Tit's theorem about incidence geometries ([14], Theorem 1).

Theorem 7.4 Suppose $X$ is a piecewise spherical (respectively Euclidean) complex of dimension $n \geq 3$ satisfying

(1) $X$ is simply connected.

(2) The link of every $k$-cell, $k \leq n-4$, is simply connected.

(3) The link of every $(n-3)$-cell is isometric to a building.

Then $X$ is isometric to a spherical building (respectively metric Euclidean building).

Proof In the spherical case, the theorem follows from Theorem 4.1 and induction. In the Euclidean case, links in $X$ are spherical buildings (by the spherical case of the theorem) so $X$ is locally $\operatorname{CAT}(0)$. Since $X$ is simply connected, it is also globally $\operatorname{CAT}(0)$ (Theorem 2.1(2)). The theorem now follows from Corollary 5.3.

Another interesting metric characterization involves extensions of geodesics. A local geodesic $\gamma$ ending at $x$ extends discretely if the set of directions in which $\gamma$ can be geodesically continued through $x$ is a non-empty discrete subset of $\mathrm{lk}(x, X)$, or equivalently, if the set of points $v \in \mathrm{k}(x, X)$ at distance $\geq \pi$ from $\gamma_{x}$ is non-empty and discrete. We say a geodesic metric space $X$ has the discrete extension property if $\gamma$ extends discretely for every local geodesic $\gamma$.

Theorem 7.5 Suppose $X$ is a connected, locally spherical (respectively Euclidean) space of dimension $n \geq 2$, and suppose

(1) $X$ is $C A T(1)$ (respectively $C A T(0)$ ),

(2) $X$ has the discrete extension property.

Then $X$ is isometric to a spherical building (respectively metric Euclidean building). 
Proof First note that if $X$ has the discrete extension property, then so does $L_{x}=\operatorname{lk}(x, X)$ for every $x$. For if $v \in L_{x}$ and $\gamma$ is a geodesic emanating from $x$ in direction $v$, then for any point $y=\gamma(t)$ in a conelike neighborhood of $x$, $L_{y}=\mathbb{S}^{0} * \mathrm{lk}\left(v, L_{x}\right)$. Thus, if every point in $L_{y}$ has a non-empty discrete set of points at distance $\geq \pi$, then the same holds in $\operatorname{lk}\left(v, L_{x}\right)$. (See the appendix of [7] for details on distances in spherical suspensions.)

The discrete extension property also implies that $L_{x}$ is connected. For if $\sigma$ is a spherical $(n-1)$-cell in $L_{x}$ and $v \in L_{x}$ is not in the connected component of $\sigma$, then all of $\sigma$ has distance $\geq \pi$ from $v$.

The theorem now follows by induction on $n$. If $n=2$, then the discrete extension property implies that $L_{x}$ is connected, has diameter $\pi$, and every vertex has valence at least 2 . Thus, by Theorem 6.1 , and the remark following it, $L_{x}$ is isometric to a 1-dimensional spherical building for all $x$. By Theorem 3.8, (respectively Corollary 5.3) $X$ is isometric to a spherical (respectively metric Euclidean) building.

If $n>2$, then $L_{x}$ is a connected, locally spherical space satisfying conditions (1) and (2) of the theorem. By induction $L_{x}$ is isometric to a spherical building for every $x$, and the conclusion follows from Theorem 3.8 (respectively Corollary $5.3)$.

\section{References}

[1] W Ballmann, Lectures on Spaces of Nonpositive Curvature, DMV Seminar 25, Birkhäuser (1995)

[2] W Ballmann, M Brin, Orbihedra of nonpositive curvature, Inst. Hautes Études Sci. Publ. Math. 82 (1996) 169-209

[3] W Ballmann, M Brin, Diameter rigidity of spherical polyhedra, Duke Math. J. 97 (1999) 235-259

[4] M Bridson, A Haefliger, Metric Spaces of Non-positive Curvature, SpringerVerlag (1999)

[5] N Bourbaki, Groupes et Algebrès de Lie, Ch. 4-6, Masson, Paris (1981)

[6] K Brown, Buildings, Springer-Verlag, New York (1989)

[7] R Charney, M W Davis, Singular metrics of nonpositive curvature on branched covers of Riemannian manifolds, Amer. J. Math. 115 (1993) 929-1009

[8] M W Davis, Buildings are CAT(0), from: "Geometry and Cohomology in Group Theory", ed. by P Kropholler, G Niblo and R Stöhr, LMS Lecture Notes Series 252, Cambridge University Press (1998) 
[9] M Gromov, Hyperbolic groups, from: "Essays in Group Theory", ed. by S M Gersten, MSRI Publ. 8, Springer-Verlag, New York (1987) 75-264

[10] W M Kantor, Generalized polygons, $S C A B$ 's and $G A B$ 's, from: "Buildings and the Geometry of Diagrams", LNM 1181, Springer-Verlag, New York (1986) 79 158

[11] B Kleiner, B Leeb, Rigidity of quasi-isometries for symmetric spaces and Euclidean buildings, Publ. IHES 86 (1997) 115-197

[12] A Neumaier, Some aporadic geometries related to PG(3,2), Arch. Math. 42 (1984) 89-96

[13] M Ronan, Lectures on Buildings, Perspectives in Mathematics, Vol. 7, Academic Press, San Diego (1989)

[14] J Tits, A local approach to buildings, from: "The Geometric Vein, Coxeter Festschrift", ed. by C Davis, B Grüenbaum and F A Sherk, Springer-Verlag, New York (1981) 519-547 